\title{
A head loss model for homogeneous slurry transport for medium sized particles
}

\author{
Sape A. Miedema \\ Delft University of Technology, Mekelweg 2, 2628 CD Delft, The Netherlands. \\ Tel.: +31-15-2788359. E-mail: s.a.miedema@tudelft.nl
}

\begin{abstract}
Slurry transport in horizontal and vertical pipelines is one of the major means of transport of sands and gravels in the dredging industry. There exist 4 main flow regimes, the fixed or stationary bed regime, the sliding bed regime, the heterogeneous flow regime and the homogeneous flow regime. Of course the transitions between the regimes are not very sharp, depending on parameters like the particle size distribution. The focus in this paper is on the homogeneous regime. Often the so called equivalent liquid model (ELM) is applied, however many researchers found hydraulic gradients smaller than predicted with the ELM, but larger that the hydraulic gradient of liquid. Talmon (2011, 2013) derived a fundamental equation (method) proving that the hydraulic gradient can be smaller than predicted by the ELM, based on the assumption of a particle free viscous sub-layer. He used a 2D velocity distribution without a concentration distribution.

In this paper 5 methods are described (and derived) to determine the hydraulic gradient in homogeneous flow, of which the last method is based on pipe flow with a concentration distribution. It appears that the use of von Driest (Schlichting, 1968) damping, if present, dominates the results, however applying a concentration distribution may neutralise this. The final equation contains both the damping and a concentration distribution giving the possibility to calibrate the constant in the equation with experimental data. The final equation is flexible and gives a good match with experimental results in vertical and horizontal pipelines for a value of $A_{C v}=1.3$. Data of horizontal experiments $D_{p}=$ $0.05-0.30 \mathrm{~m}, d=0.04 \mathrm{~mm}$, vertical experiments $D_{p}=0.026 \mathrm{~m}, d=0.125,0.345,0.560$, and $0.750 \mathrm{~mm}$.
\end{abstract}

Keywords: Slurry transport; Homogeneous transport; Viscous sub layer; Mixing length.

\section{INTRODUCTION}

Slurry transport in horizontal and vertical pipelines is one of the major means of transport of sands and gravels in the dredging industry. There exist 4 main flow regimes, the fixed or stationary bed regime, the sliding bed regime, the heterogeneous flow regime and the homogeneous flow regime. Of course the transitions between the regimes are not very sharp, depending on parameters like the particle size distribution. In the case of very fine particles and/or very high line speeds, the mixture is often considered to be a liquid with the liquid density $\rho_{l}$ equal to the mixture density $\rho_{m}$, where the liquid density $\rho_{l}$ can be replaced by the mixture density $\rho_{m}$ in the hydraulic gradient equations. The velocity profile in a cross section of the pipe is considered to be symmetrical and the slip between the particles and the liquid is considered negligible. The concentration is assumed to be uniform over the cross section. Thus, the transport (or delivered) concentration and the spatial concentration are almost equal and will be named $C_{v}$. This is often referred to as the equivalent liquid model (ELM).

Since the pressure losses are often expressed in terms of the hydraulic gradient, first some basic equations for the hydraulic gradient and the relative excess hydraulic gradient (solids effect) are given. The hydraulic gradient according to the equivalent liquid model is:

$i_{m}=\frac{\Delta p_{m}}{\rho_{l} \cdot g \cdot \Delta L}=\frac{\lambda_{m} \cdot v_{l s}^{2}}{2 \cdot g \cdot D_{p}} \cdot \frac{\rho_{m}}{\rho_{l}}$

with: $\quad \lambda_{m}=\lambda_{l}$ where it is assumed that the Darcy-Weisbach friction factors for liquid $\lambda_{l}$ and mixture $\lambda_{m}$ are equal. This can also be written as:

$i_{m}=i_{l} \cdot\left(1+R_{s d} \cdot C_{v}\right)$

Newitt et al. (1955) found that only $60 \%$ of the solids weight should contribute to the mixture density in order to obtain the equivalent liquid model, but this depends on the line speed and possibly on other parameters as well. Many others also found hydraulic gradients below the ELM at high line speeds. Wilson et al. (1992) explain this with the effect of near wall lift resulting in an almost particle free viscous sub layer. However for very small particles values are found giving a higher value of the pressure gradient, which is often explained by correcting (increase) the apparent kinematic viscosity, for example with the Thomas (1965) equation.

The pressure losses can be shown in an almost dimensionless form in a double logarithmic graph with the relative excess hydraulic gradient $E_{r h g}$ as the ordinate and the hydraulic liquid gradient $i_{l}$ as the abscissa. In terms of the relative excess hydraulic gradient, $E_{r h g}$, the above equation can be written as:

$E_{\text {rhg }}=\frac{i_{m}-i_{l}}{R_{s d} \cdot C_{v}}=\frac{\lambda_{l} \cdot v_{l s}^{2}}{2 \cdot g \cdot D_{p}}=i_{l}$

So in the $E_{r h g}\left(i_{l}\right)$ graph the above equation results in a straight line giving $E_{r h g}=i_{l}$. Fig. 1 shows experimental data of Thomas (1976) of $d=0.04 \mathrm{~mm}$ iron ore in a $D_{p}=0.1585 \mathrm{~m}$ horizontal pipe versus the Delft Head Loss \& Limit Deposit Velocity (DHLLDV) model, where the 4 term Thomas (1965) viscosity equation and the homogeneous flow correction equation (37) with $A_{C v}=1.3$ are implemented. The match is remarkable. 


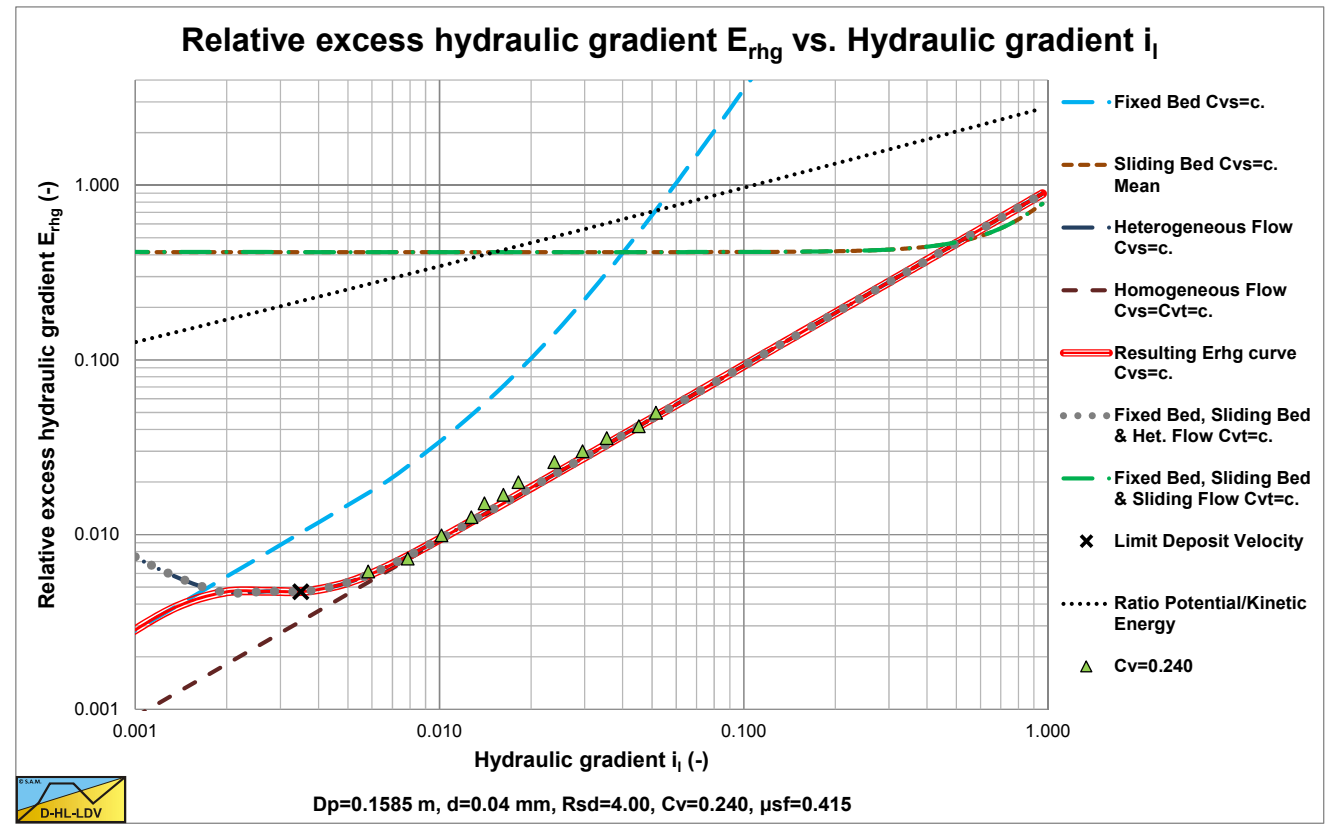

Fig. 1. The Thomas (1976) experimental data in a $E_{r h g}\left(i_{l}\right)$ graph.

\section{APPROACH}

In order to test the Talmon $(2011,2013)$ method and to check if there are alternative methods the following approach is followed:

1. Method 1: First the Talmon $(2011,2013)$ method is discussed briefly.

2. Method 2: Since the Talmon $(2011,2013)$ method uses a 2D approach, with von Driest damping (Schlichting, 1968), but without a real concentration profile, in this second method the equations are derived for pipe flow with the Nikuradse (1933) mixing length equation, without von Driest damping (Schlichting, 1968) and without a real concentration profile. The results are corrected for the volume flow.

3. Method 3: Von Driest damping (Schlichting, 1968) is added to method 2 , resulting in a velocity profile comparable and very close to the Talmon $(2011,2013)$ method 1 . So method 1 and method 3 are equivalent.

4. Method 4: The "Law of the Wall" 2D approach without von Driest damping (Schlichting, 1968).

5. The 4 methods are compared and an equation describing the average behavior is derived.

6. Method 5: Finally a concentration profile is added to method 2. This method can simulate all previous methods, depending on the concentration profile chosen.

7. Based on experiments a value for the parameters of the concentration profile is chosen.

\section{METHOD 1: THE TALMON (2011) \& (2013) HOMOGENEOUS REGIME EQUATION}

Talmon $(2011,2013)$ derived an equation to correct the homogeneous equation (the ELM model) for the slurry density, based on the hypothesis that the viscous sub-layer hardly contains solids at very high line speeds in the homogeneous regime. This theory results in a reduction of the resistance compared with the ELM, but the resistance is still higher than the resistance of clear liquid. Talmon $(2011,2013)$ used the Prandtl approach for the mixing length, which is a 2D approach for open channel flow with a free surface.
The Prandtl approach was extended with damping near the wall to take into account the viscous effects near the wall, according to von Driest (Schlichting, 1968). The Talmon (2011, 2013) approach resulted in the following equation, with $\alpha_{h}=$ 6.7:

$$
\begin{aligned}
& \frac{\lambda_{m}}{\lambda_{l}}=\frac{1}{\left(\alpha+\sqrt{\frac{\lambda_{l}}{8}} \cdot R_{s d} \cdot C_{v}+1\right)^{2}} \text { and } \\
& E_{r h g}=i_{l} \cdot \frac{R_{s d} \cdot C_{v}+1-\left(\alpha \cdot \sqrt{\frac{\lambda_{l}}{8}} \cdot R_{s d} \cdot C_{v}+1\right)^{2}}{R_{s d} \cdot C_{v} \cdot\left(\alpha_{h} \cdot \sqrt{\frac{\lambda_{l}}{8}} \cdot R_{s d} \cdot C_{v}+1\right)^{2}}=\alpha_{E} \cdot i_{l}
\end{aligned}
$$

This equation underestimates the hydraulic gradient (overestimates the effect of a particle free viscous sub layer) in a number of cases (small and large particles) as Talmon (2011, 2013) proves with the examples shown in his papers. Only for $d_{50}=0.37 \mathrm{~mm}$ and $D_{p}=0.15 \mathrm{~m}$ (medium particles) there is a good match. The philosophy behind this theory, combining a viscous sub-layer with liquid with a kernel with mixture, is however very interesting, because it explains fundamentally why the hydraulic gradient can be lower than the hydraulic gradient according to the ELM, as has been shown by many researchers.

The model has been derived using the standard mixing length equation for 2D flow and without a concentration distribution. When reproducing this method it was found that the coefficient $\alpha_{h}$ is not a constant but this coefficient depends on the value of $R_{s d} \cdot C_{v}$ according to Fig. 2 . The value of 6.7 is found for a value of about 0.6 of the abscissa.

\section{METHOD 2: THE APPROACH USING THE NIKURADSE (1933) MIXING LENGTH}

The concept of Talmon $(2011,2013)$ is adopted, but modified by using pipe flow with the Prandtl (1925) and Nikuradse (1933) 


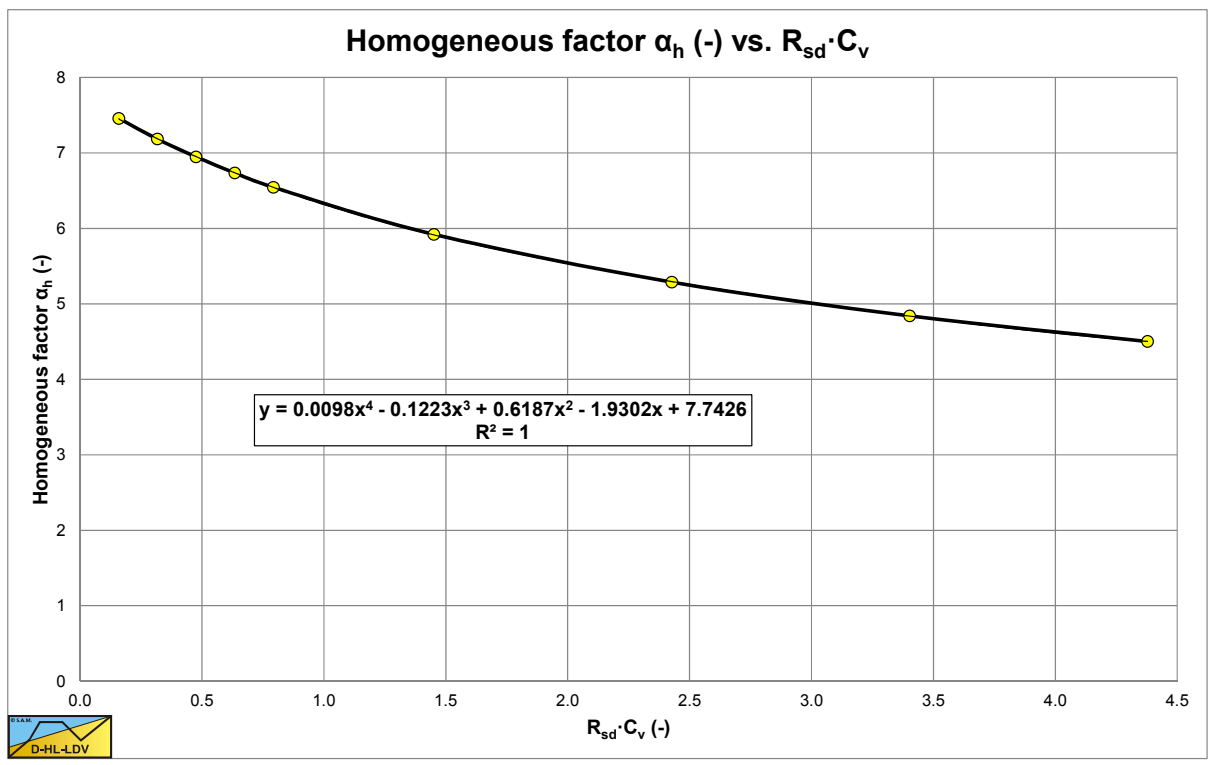

Fig. 2. The coefficient $\alpha_{h}$ as a function of $R_{s d} \cdot C_{v}$ for method 1.

mixing length equations, a linear shear stress distribution with a maximum at the pipe wall and zero in the center and a concentration distribution, assuming that in the homogeneous regime the mixture can be considered a Newtonian liquid with properties slightly different from those of water. The shear stress between the mixture, the slurry, and the pipe wall is the sum of the viscous shear stress and the turbulent shear stress:

$$
\begin{aligned}
& \tau=\tau_{v}+\tau_{t}=\mu_{v} \cdot \frac{\partial u}{\partial z}+\mu_{t} \cdot \frac{\partial u}{\partial z} \\
& =\rho_{m} \cdot v_{m} \cdot \frac{\partial u}{\partial z}+\rho_{m} \cdot v_{t} \cdot \frac{\partial u}{\partial z} \\
& \text { with: } \quad v_{t}=\ell^{2} \cdot\left|\frac{\partial u}{\partial z}\right|
\end{aligned}
$$

Now the shear stress can be expressed as (with $z$ the distance from the pipe wall):

$$
\begin{aligned}
& \tau=\rho_{m} \cdot\left(u_{*}\right)^{2} \cdot\left(\frac{R-z}{R}\right) \\
& =\rho_{m} \cdot\left(v_{m}+\ell^{2} \cdot\left|\frac{\partial u}{\partial z}\right|\right) \cdot \frac{\partial u}{\partial z}
\end{aligned}
$$

with: $R=D_{p} / 2$

This is a second degree function of the velocity gradient. Solving this with respect to the velocity gradient gives:

$$
\begin{gathered}
\frac{\partial u}{\partial z}=\frac{-\frac{\mu_{m}}{\rho_{m}}+\sqrt{\left(\frac{\mu_{m}}{\rho_{m}}\right)^{2}+4 \cdot \ell^{2} \cdot\left(u_{*}\right)^{2} \cdot\left(\frac{R-z}{R}\right)}}{2 \cdot \ell^{2}} \\
=\frac{2 \cdot\left(u_{*}\right)^{2} \cdot\left(\frac{R-z}{R}\right)}{\frac{\mu_{m}}{\rho_{m}}+\sqrt{\left(\frac{\mu_{m}}{\rho_{m}}\right)^{2}+4 \cdot \ell^{2} \cdot\left(u_{*}\right)^{2} \cdot\left(\frac{R-z}{R}\right)}}
\end{gathered}
$$

A required condition for pipe flow is, that the integral of the velocity over the pipe cross-section equals the average line speed times the cross-section, so:

$\int_{0}^{R} \frac{\partial u}{\partial z} \cdot 2 \cdot \pi \cdot(R-z) \cdot d z=v_{l s} \cdot \pi \cdot R^{2}$

The Nikuradse (1933) equation for the mixing length in pipe flow for large Reynolds numbers is:

$$
\begin{aligned}
& \frac{\ell}{R}=0.14-0.08 \cdot\left(1-\frac{z}{R}\right)^{2}-0.06 \cdot\left(1-\frac{z}{R}\right)^{4} \\
& =0.14 \cdot\left(1-\frac{4}{7} \cdot\left(1-\frac{z}{R}\right)^{2}-\frac{3}{7} \cdot\left(1-\frac{z}{R}\right)^{4}\right)
\end{aligned}
$$

The velocity profile can be determined by numerical integration. This velocity profile however, should result in an average velocity equal to the line speed used to determine the friction velocity. This appeared to be valid for very high line speeds (Reynolds numbers) in the range of 1300-1500 m/s, which is far above the range dredging companies operate (3-10 $\mathrm{m} / \mathrm{s})$. For line speeds in the range $3-10 \mathrm{~m} / \mathrm{s}$, the velocity profile resulted in an average velocity smaller than the line speed with a factor $0.8-1.0$. Introducing a factor $\beta$ and an extra term in the mixing length equation solves this problem. The original Nikuradse (1933) equation is multiplied with a factor $\beta$ and an extra term is added to ensure that $\ell / R=\kappa \cdot z$ for $z=0$, like is the case in the original equation. The factor $\beta$ is determined for each calculation in such a way, that the flow following from the line speed times the cross section of the pipe, equals the flow from integration of the velocity profile.

$$
\begin{aligned}
\frac{\ell}{R}=\beta \cdot\left(\begin{array}{l}
0.14-0.08 \cdot\left(1-\frac{z}{R}\right)^{2} \\
-0.06 \cdot\left(1-\frac{z}{R}\right)^{4}
\end{array}\right) \\
+(1-\beta) \cdot \kappa \cdot \frac{z}{R} \cdot e^{-\frac{z}{0.00002 \cdot R}}
\end{aligned}
$$




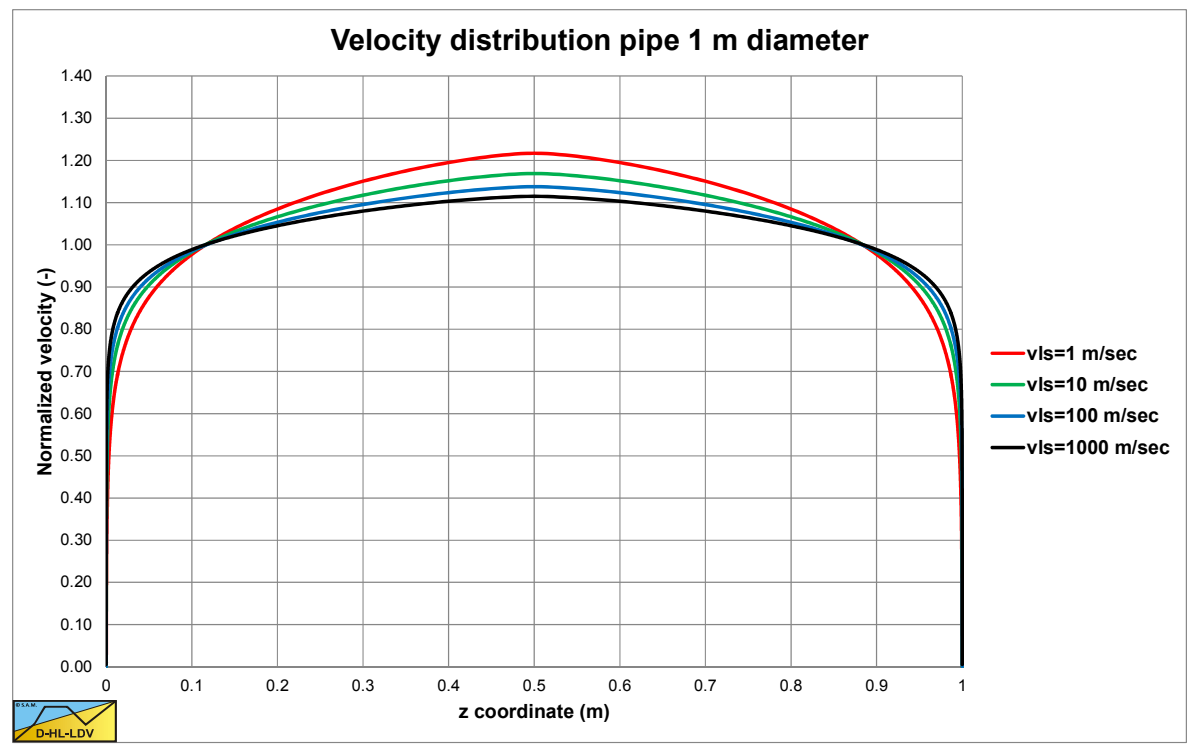

Fig. 3. The resulting velocity distributions in a $D_{p}=1 \mathrm{~m}$ pipe, corrected for the flow.

Fig. 3 shows the resulting velocity distributions for a $1 \mathrm{~m}$ diameter pipe. Now the concept is, that a mixture flow with liquid as a carrier liquid in the viscous sub layer will have a lower resistance than a mixture flow with mixture in the viscous sub-layer. One can also say that in order to get the same pipeline resistance, the velocity in the center of the pipe of mixture with liquid in the viscous sub-layer $u_{m}$ has to be higher than the case with mixture or liquid in the whole pipe $u_{l}$. Assuming that the dynamic viscosity of the mixture is equal to the dynamic viscosity of the carrier liquid, $\mu_{m}=\mu_{l}$, in the viscous sub-layer and the boundary layer where no solids are present, gives:

$\frac{\partial u}{\partial z}=\frac{-\frac{\rho_{l}}{\rho_{m}} \cdot v_{l}+\sqrt{\left(\frac{\rho_{l}}{\rho_{m}} \cdot v_{l}\right)^{2}+4 \cdot \ell^{2} \cdot\left(u_{*}\right)^{2} \cdot\left(\frac{R-z}{R}\right)}}{2 \cdot \ell^{2}}$

Now assuming that the term with the density ratio is relevant only near the pipe wall and not in the center of the pipe, this equation will simulate a mixture with liquid in the viscous sublayer. In fact, the density ratio reduces the effect of the kinematic viscosity, which mainly affects the viscous sub-layer. The velocity difference in the center of the pipe between mixture and liquid, $u_{m}-u_{l}$, can now be determined with:

$$
\begin{gathered}
u_{m}-u_{l}=\int_{0}^{R}\left(\frac{\partial u}{\partial z}\right)_{m} \cdot d z-\int_{0}^{R}\left(\frac{\partial u}{\partial z}\right)_{l} \cdot d z \\
=\int_{0}^{R} \frac{\rho_{l} \frac{\rho_{m}}{\rho_{l}}+\sqrt{\left(\frac{\rho_{l}}{\rho_{m}} \cdot v_{l}\right)^{2}+4 \cdot \ell^{2} \cdot(u *)^{2} \cdot\left(\frac{R-z}{R}\right)}}{2 \cdot \ell^{2}} \cdot d z \\
-\int_{0}^{R} \frac{v_{l}+\sqrt{\left(v_{l}\right)^{2}+4 \cdot \ell^{2} \cdot(u *)^{2} \cdot\left(\frac{R-z}{R}\right)}}{2 \cdot \ell^{2}} \cdot d z
\end{gathered}
$$

This velocity difference, in the center of the pipe, is about equal to the difference of the average line speeds, however both can be determined numerically. Further it appears from the numerical solution of this equation, that dividing the velocity difference by the average liquid velocity $u_{l}$ or $v_{l s, l}$ results in a factor $F$, which only depends on the volumetric concentration $C_{v}$, the relative submerged density $R_{s d}$ and slightly on the line speed $v_{l s}$ in the range $3-10 \mathrm{~m} / \mathrm{s}$ and on the pipe diameter $D_{p}$ through the Darcy-Weisbach friction factor $\lambda_{l}$, according to:

$$
\begin{aligned}
& F=\frac{u_{m}-u_{l}}{u_{l}}=\frac{v_{l s, m}-v_{l s, l}}{v_{l s, l}}=\alpha_{h} \cdot \lambda_{l} \cdot R_{s d} \cdot C_{v} \\
& =\alpha_{h} \cdot \lambda_{l} \cdot\left(\frac{\rho_{m}}{\rho_{l}}-1\right)
\end{aligned}
$$

The shear stress at the pipe wall of a Newtonian liquid is by definition:

$\rho_{l} \cdot\left(u_{*}\right)^{2}=\frac{\lambda_{l}}{8} \cdot \rho_{l} \cdot v_{l s, l}^{2}$

and

$\rho_{m} \cdot\left(u_{*}\right)^{2}=\frac{\lambda_{m}}{8} \cdot \rho_{m} \cdot v_{l s, m}^{2}$

From this a relation for the ratio of the Darcy-Weisbach friction coefficients of a flow with mixture in the center and carrier liquid in the viscous sub-layer to a flow with $100 \%$ liquid can be derived.

$$
\begin{aligned}
& \frac{\lambda_{l}}{8} \cdot v_{l s, l}^{2}=\frac{\lambda_{m}}{8} \cdot v_{l s, m}^{2} \\
& \Rightarrow \quad \frac{\lambda_{m}}{\lambda_{l}}=\frac{v_{l s, l}^{2}}{v_{l s, m}^{2}} \\
& \Rightarrow \quad \frac{\lambda_{m}}{\lambda_{l}}=\frac{v_{l s, l}^{2}}{\left(F \cdot v_{l s, l}+v_{l s, l}\right)^{2}}=\frac{1}{(F+1)^{2}}
\end{aligned}
$$

Equation (15) is independent of the method used, but the factor $F$ is. Substituting the factor $F$ from equation (13) gives:

$$
\frac{\lambda_{m}}{\lambda_{l}}=\frac{1}{(F+1)^{2}}=\frac{1}{\left(\alpha_{h} \cdot \lambda_{l} \cdot R_{s d} \cdot C_{v}+1\right)^{2}}
$$


This ratio depends on the homogeneous factor $\alpha_{h}$, the Darcy-Weisbach friction factor $\lambda_{l}$, the volumetric concentration $C_{v}$ and the relative submerged density $R_{s d}$. The ratio of the hydraulic gradients is now:

$$
\begin{aligned}
& \frac{i_{m}}{i_{l}}=\frac{\lambda_{m} \cdot \rho_{m}}{\lambda_{l} \cdot \rho_{l}}=\frac{1+R_{s d} \cdot C_{v}}{\left(\alpha_{h} \cdot \lambda_{l} \cdot R_{s d} \cdot C_{v}+1\right)^{2}} \\
& \Rightarrow \quad i_{m}=i_{l} \cdot \frac{1+R_{s d} \cdot C_{v}}{\left(\alpha_{h} \cdot \lambda_{l} \cdot R_{s d} \cdot C_{v}+1\right)^{2}}
\end{aligned}
$$

This gives for the excess hydraulic gradient $i_{m}-i_{l}$ (the solids effect):

$$
\begin{aligned}
i_{m}-i_{l}= & i_{l} \cdot \frac{1+R_{s d} \cdot C_{v}}{\left(\alpha_{h} \cdot \lambda_{l} \cdot R_{s d} \cdot C_{v}+1\right)^{2}} \\
& -i_{l} \cdot \frac{\left(\alpha_{h} \cdot \lambda_{l} \cdot R_{s d} \cdot C_{v}+1\right)^{2}}{\left(\alpha_{h} \cdot \lambda_{l} \cdot R_{s d} \cdot C_{v}+1\right)^{2}} \\
= & i_{l} \cdot \frac{1+R_{s d} \cdot C_{v}-\left(\alpha_{h} \cdot \lambda_{l} \cdot R_{s d} \cdot C_{v}+1\right)^{2}}{\left(\alpha_{h} \cdot \lambda_{l} \cdot R_{s d} \cdot C_{v}+1\right)^{2}}
\end{aligned}
$$

The relative excess hydraulic gradient $E_{r h g}$ is now:

$$
\begin{aligned}
E_{r h g} & =\frac{i_{m}-i_{l}}{R_{s d} \cdot C_{v}} \\
& =i_{l} \cdot \frac{1+R_{s d} \cdot C_{v}-\left(\alpha_{h} \cdot \lambda_{l} \cdot R_{s d} \cdot C_{v}+1\right)^{2}}{R_{s d} \cdot C_{v} \cdot\left(\alpha_{h} \cdot \lambda_{l} \cdot R_{s d} \cdot C_{v}+1\right)^{2}} \\
& =\alpha_{E} \cdot i_{l}
\end{aligned}
$$

The limiting value for the relative excess hydraulic gradient $E_{r h g}$ for a volumetric concentration $C_{v}$ approaching zero, becomes:

$$
E_{r h g}=\frac{i_{m}-i_{l}}{R_{s d} \cdot C_{v}}=i_{l} \cdot\left(1-2 \cdot \alpha_{h} \cdot \lambda_{l}\right)
$$

For sand and gravel with a density of $2.65 \mathrm{ton} / \mathrm{m}^{3}$, the factor $\alpha_{h}$ is about 9.3, almost independent of the pipe diameter $D_{p}$ and the line speed $v_{l s}$ for pipes with diameters of $0.5 \mathrm{~m}$ up to $1.2 \mathrm{~m}$ and line speeds from $2 \mathrm{~m} / \mathrm{s}$ up to $10 \mathrm{~m} / \mathrm{s}$. For very small pipes and very low line speeds, like $D_{p}=0.1 \mathrm{~m}$ and $v_{l s}=1 \mathrm{~m} / \mathrm{s}$, this factor decreases to about 8.5. The factor $\alpha_{h}$ is not $100 \%$ linear with the term $R_{s d} \cdot C_{v}$ for sands with a density of $2.65 \mathrm{ton} / \mathrm{m}^{3}$ and volumetric concentrations up to $35-40 \%$. Since the solution depends on $R_{s d} \cdot C_{v}$ combined, the factor $\alpha_{h}$ also depends on this and not on $R_{s d}$ and $C_{v}$ separately. Fig. 4 shows the dependency of the factor $\alpha_{h}$ on the relative excess density $R_{s d} \cdot C_{v}$. The factor $\alpha_{E}$ decreases with increasing concentration and relative submerged density of the solids and increases with increasing line speed. At normal line speeds $(3-6 \mathrm{~m} / \mathrm{s})$ and concentrations $(0.1-0.3)$ this factor is about $0.74-0.78$ (see Fig. 9). A larger pipe gives less reduction. This is caused by the smaller DarcyWeisbach friction coefficient $\lambda_{l}$ of larger pipes.

\section{METHOD 3: ADDING THE VON DRIEST DAMPING TO METHOD 2}

Talmon (2013) used the Prandtl approach for the mixing length, which is a 2D approach for open channel flow with a free surface. The Prandtl approach was extended with damping near the wall to take into account the viscous effects near the wall, according to von Driest (Schlichting, 1968):

Prandl: $\quad \ell=\kappa \cdot z$

von Driest : $\ell=\kappa \cdot z \cdot\left(1-e^{-z^{+} / A}\right)$

with : $\quad \mathrm{z}^{+}=\frac{z \cdot u_{*}}{v_{f l}} \quad \mathrm{~A}=26$

Fig. 7 and Fig. 5 show the velocity profile and the mixing length profile of the Talmon (2013) approach with von Driest (Schlichting, 1968) damping and the Nikuradse (1933) approach without damping. In both cases, the mixing length equations have been corrected in order to get the correct volume flow. There is a clear difference of the velocity profiles.

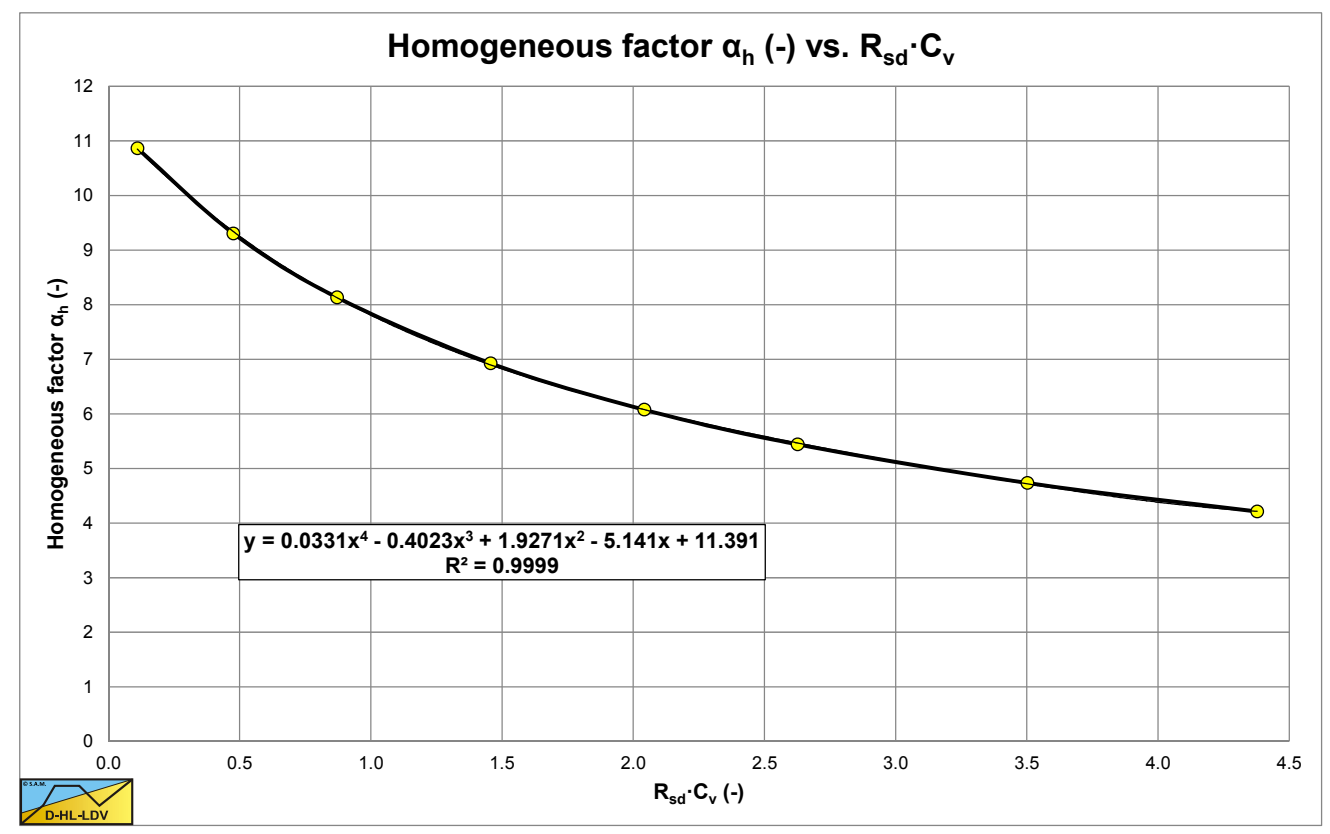

Fig. 4. The homogeneous factor $\alpha_{h}$ as a function of $R_{s d} \cdot C_{v}$ for method 2 . 


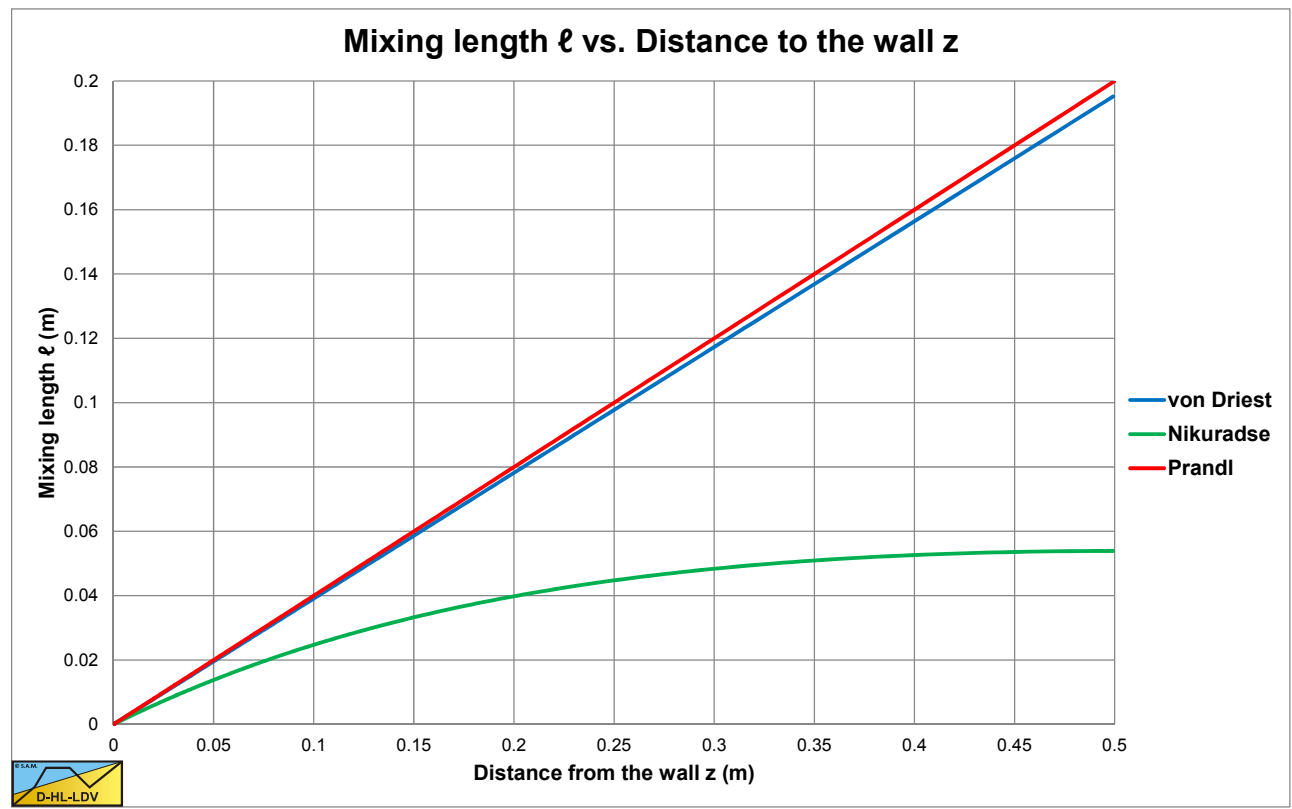

Fig. 5. The mixing length versus the distance to the wall for a $D_{p}=1 \mathrm{~m}$ pipe at a line speed $v_{l s}=5 \mathrm{~m} / \mathrm{s}, \kappa_{\text {Prandtl }}=0.4, \kappa_{\text {vonDriest }}=0.3915$, and $\kappa_{\text {Nikuradse }}=0.4$, without von Driest (Schlichting, 1968) damping.

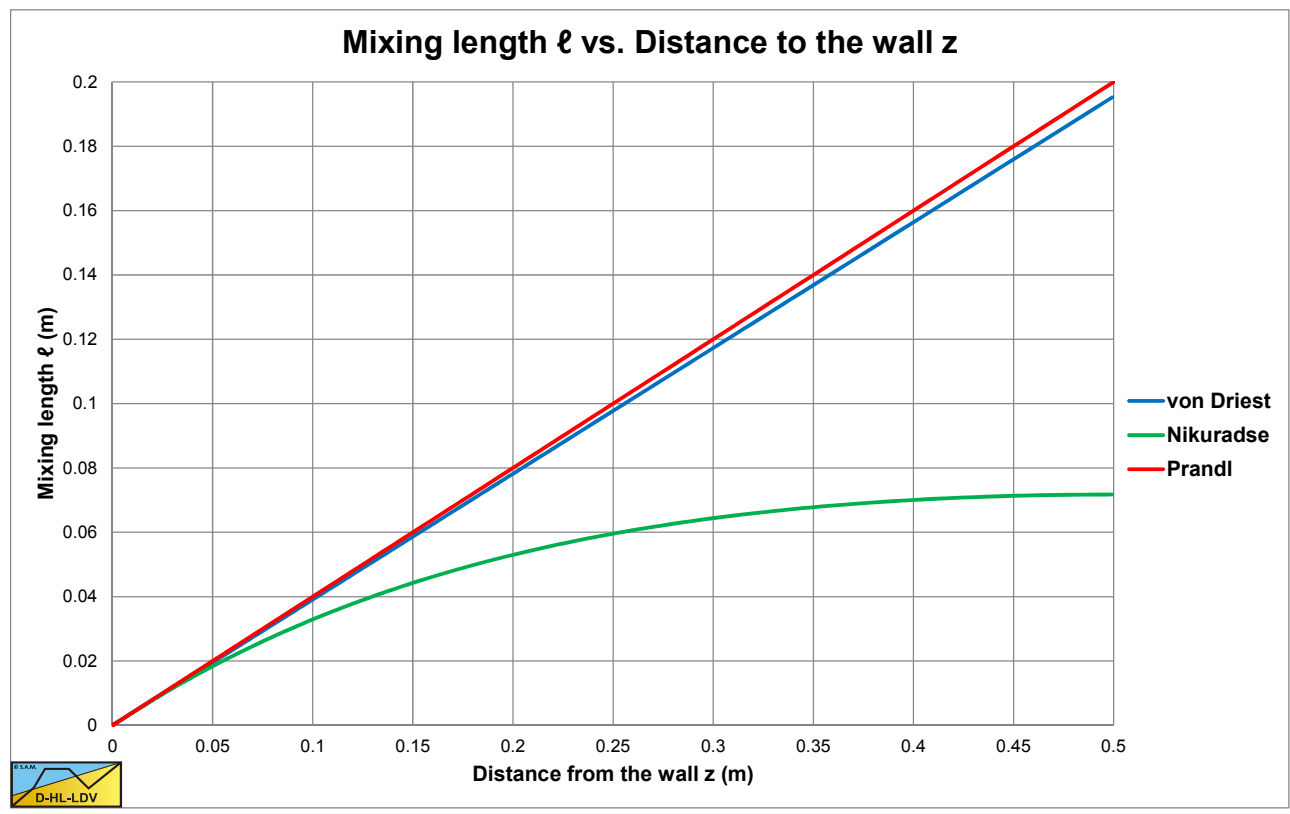

Fig. 6. The mixing length versus the distance to the wall for a $D_{p}=1 \mathrm{~m}$ pipe at a line speed $v_{l s}=5 \mathrm{~m} / \mathrm{s}, \kappa_{\text {Prandtl }}=0.4$, $\kappa_{\text {vonDriest }}=0.3915$, and $\kappa_{\text {Nikuradse }}=0.4$, with von Driest (Schlichting, 1968) damping.

Applying the von Driest (Schlichting, 1968) damping to the Nikuradse (1933) equation (9) for the mixing length in pipe flow for large Reynolds numbers according to:

$$
\begin{aligned}
\frac{\ell}{R}= & 0.14 \cdot\left(1-\frac{4}{7} \cdot\left(1-\frac{z}{R}\right)^{2}-\frac{3}{7} \cdot\left(1-\frac{z}{R}\right)^{4}\right) \\
& \cdot\left(1-e^{-z^{+} / A}\right)
\end{aligned}
$$

Gives almost exactly the same results as the Talmon (2013) approach, although the mixing length is completely different as is shown in Fig. 6. Only very close to the wall, where the viscous effects dominate, the same mixing lengths are found. Apparently, the von Driest damping, effective close to the pipe wall, dominates the effect of a particle free viscous sub layer, as expected. The results are almost independent of the pipe diameter $D_{p}$ and the line speed $v_{l s}$. Fig. 8 shows that the velocity profiles determined with equation (21) (Talmon) and equation (22) (Miedema) are almost the same and the behavior with respect to the hydraulic gradient reduction is equivalent.

\section{METHOD 4: THE LAW OF THE WALL APPROACH}

Often for open channel flow the so called "Law of the Wall" equations are used. Since in dredging the pipe wall is assumed to be smooth due to the continuous sanding of the pipe wall, the smooth wall approach is discussed here. Based on the following assumption for the mixing length by Prandtl (1925) and the assumption that the viscous shear stress is negligible in the 
turbulent region, the famous logarithmic velocity equation, "Law of the Wall" for the turbulent flow is derived:

$$
\begin{aligned}
& \tau=\tau_{\text {wall }} \cdot\left(1-\frac{z}{R}\right)=\rho_{l} \cdot \ell^{2} \cdot\left(\frac{d u}{d z}\right)^{2} \\
& \text { and } \ell=\kappa \cdot z \cdot\left(1-\frac{z}{R}\right)^{0.5}
\end{aligned}
$$

This "Law of the Wall" is also a 2D approach for open channel flow and does not correct for pipe flow.

The general equation for the velocity profile as a function of the distance to the smooth wall is:

$u(z)=\frac{u *}{\kappa} \cdot \ln \left(\frac{z}{z_{0}}\right)$

with: $z_{0}=0.11 \cdot \frac{v_{l}}{u_{*}}$

For the $100 \%$ liquid (or mixture) the velocity profile is defined as:

$u_{l}(z)=\frac{u_{*}}{\kappa} \cdot \ln \left(\frac{z}{z_{0, l}}\right)$

with: $z_{0, l}=0.11 \cdot \frac{v_{l}}{u_{*}}$

For the mixture with liquid in the viscous sub-layer the velocity profile can be defined as:

$u_{m}(z)=\frac{u_{*}}{\kappa} \cdot \ln \left(\frac{z}{z_{0, m}}\right)$

with: $\quad z_{0, m}=0.11 \cdot \frac{v_{l}}{u_{*}} \cdot \frac{\rho_{l}}{\rho_{m}}$
The velocity difference at the center of the pipe is now:

$$
\begin{aligned}
& u_{m}(R)-u_{l}(R)=\frac{u_{*}}{\kappa} \cdot \ln \left(\frac{\rho_{m}}{\rho_{l}}\right) \\
& =\frac{u_{*}}{\kappa} \cdot \ln \left(\frac{R}{z_{0, m}}\right)-\frac{u_{*}}{\kappa} \cdot \ln \left(\frac{R}{z_{0, l}}\right) \\
& =\frac{u_{*}}{\kappa} \cdot \ln \left(\frac{R \cdot u_{*}}{0.11 \cdot v_{l}} \cdot \frac{\rho_{m}}{\rho_{l}}\right)-\frac{u_{*}}{\kappa} \cdot \ln \left(\frac{R \cdot u_{*}}{0.11 \cdot v_{l}}\right)
\end{aligned}
$$

This gives for the Darcy-Weisbach friction coefficient ratio:

$$
\begin{aligned}
& \frac{\lambda_{m}}{\lambda_{l}}=\frac{v_{l s, l}^{2}}{v_{l s, m}^{2}}=\frac{u_{l}^{2}}{u_{m}^{2}}=\frac{u_{l}}{\left(\frac{u_{*}}{\kappa} \cdot \ln \left(\frac{\rho_{m}}{\rho_{l}}\right)+u_{l}\right)^{2}} \\
& =\frac{1}{\left(\frac{1}{\kappa} \cdot \ln \left(\frac{\rho_{m}}{\rho_{l}}\right) \cdot \sqrt{\frac{\lambda_{l}}{8}}+1\right)^{2}} \\
& \text { with: } \quad \mathrm{u}_{*}=\sqrt{\frac{\lambda_{l}}{8}} \cdot u_{l}
\end{aligned}
$$

The relative excess hydraulic gradient $E_{r h g}$ is now:

$$
\begin{aligned}
& E_{r h g}=\frac{i_{m}-i_{l}}{R_{s d} \cdot C_{v}}=\alpha_{E} \cdot i_{l} \\
& =i_{l} \cdot \frac{1+R_{s d} \cdot C_{v}-\left(\frac{1}{\kappa} \cdot \ln \left(\frac{\rho_{m}}{\rho_{l}}\right) \cdot \sqrt{\frac{\lambda_{l}}{8}}+1\right)^{2}}{R_{s d} \cdot C_{v} \cdot\left(\frac{1}{\kappa} \cdot \ln \left(\frac{\rho_{m}}{\rho_{l}}\right) \cdot \sqrt{\frac{\lambda_{l}}{8}}+1\right)^{2}}
\end{aligned}
$$

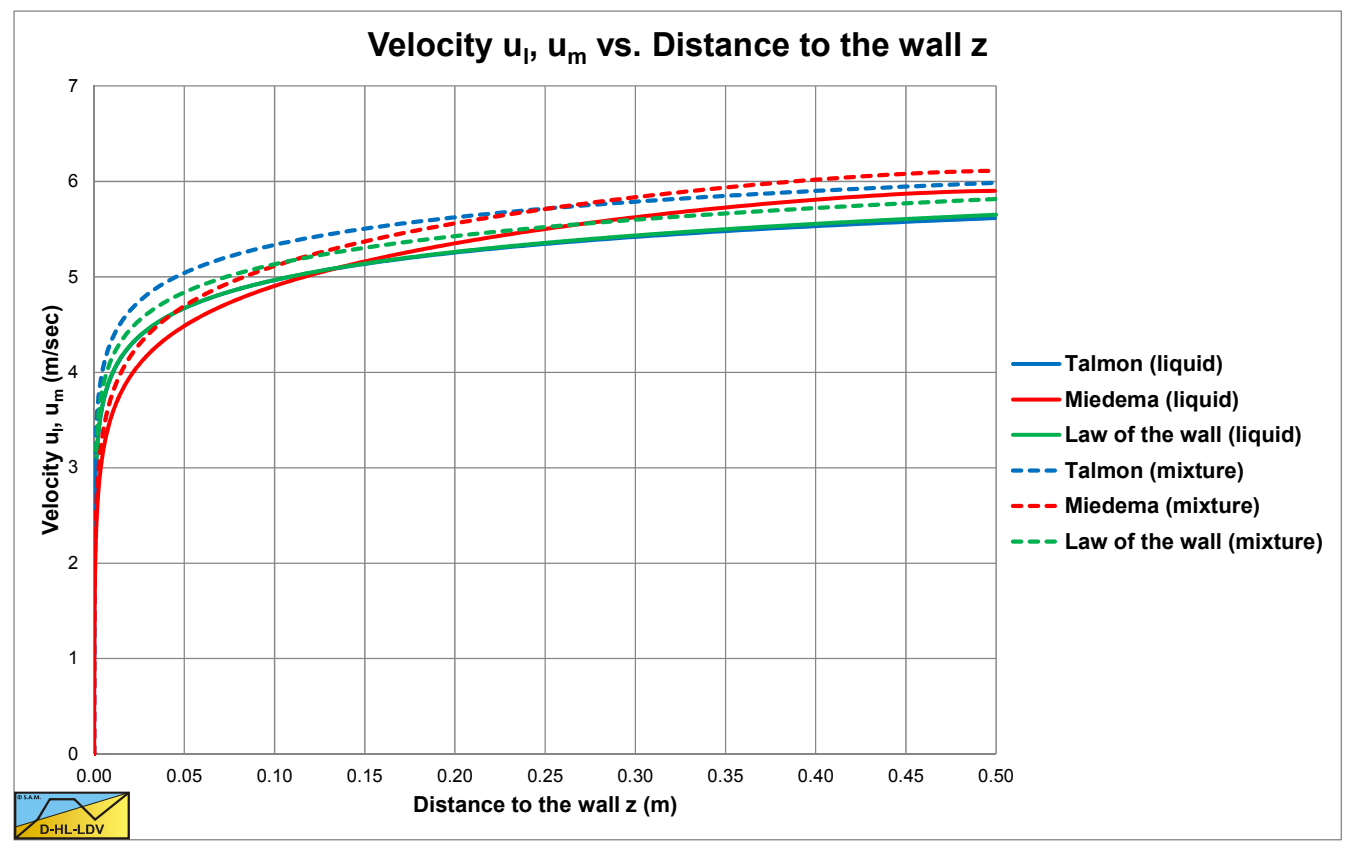

Fig. 7. The velocity versus the distance to the wall for a $D_{p}=1 \mathrm{~m}$ pipe at a line speed $v_{l s}=5 \mathrm{~m} / \mathrm{s}, \kappa_{\text {Prandtl }}=0.4, \kappa_{\text {vonDriest }}=0.3915$, and $\kappa_{\text {Nikuradse }}=0.4$, without von Driest (Schlichting, 1968) damping. 


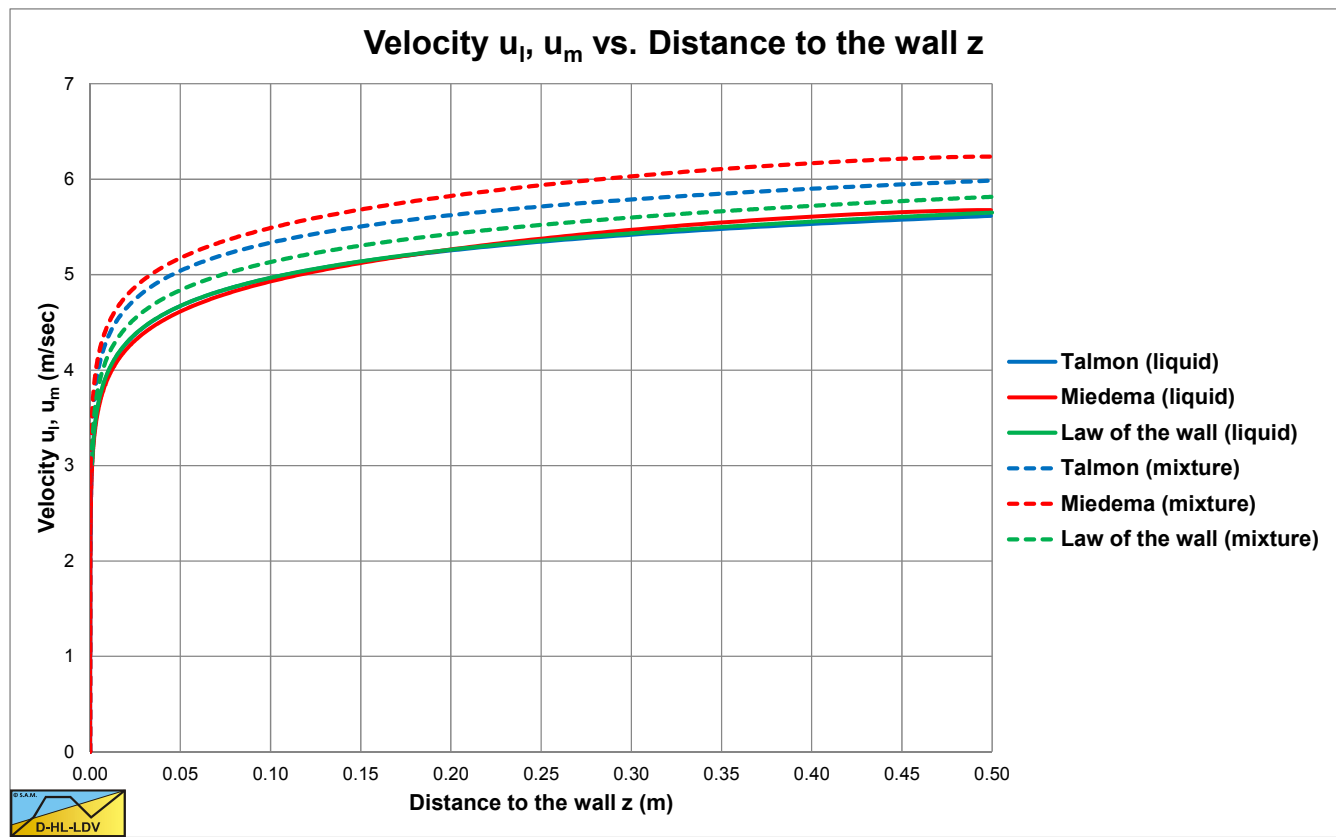

Fig. 8. The velocity versus the distance to the wall for a $D_{p}=1 \mathrm{~m}$ pipe at a line speed $v_{l s}=5 \mathrm{~m} / \mathrm{s}, \kappa_{\text {Prandtl }}=0.4, \kappa_{\text {vonDriest }}=0.3915$, and $\kappa_{\text {Nikuradse }}=0.4$, with von Driest (Schlichting, 1968) damping.

\section{COMPARISON OF THE MODELS}

Now 3 formulations are found for the reduction of the DarcyWeisbach friction factor and the relative excess hydraulic gradient $E_{r h g}$ for slurry transport of a mixture with pure carrier liquid (water) in the viscous sub-layer, these are equations (4), (16) \& (19) and (28) \& (29):

Law of the Wall (no damping)

Miedema (28)

$$
\begin{gathered}
\frac{\lambda_{m}}{\lambda_{l}}=\frac{1}{\left(\frac{1}{\kappa} \cdot \ln \left(1+R_{s d} \cdot C_{v}\right) \cdot \sqrt{\frac{\lambda_{l}}{8}}+1\right)^{2}} \\
\kappa=0.4
\end{gathered}
$$

$$
\begin{aligned}
& \frac{\lambda_{m}}{\lambda_{l}}=\frac{1}{\left(\alpha_{h} \cdot \lambda_{l} \cdot R_{s d} \cdot C_{v}+1\right)^{2}} \\
& \alpha_{h}=9.3
\end{aligned}
$$

Prandl - von Driest (damping)

Talmon (4)

$$
\begin{gathered}
\frac{\lambda_{m}}{\lambda_{l}}=\frac{1}{\left(\alpha_{h} \cdot \sqrt{\frac{\lambda_{l}}{8}} \cdot R_{s d} \cdot C_{v}+1\right)^{2}} \\
\alpha_{h}=6.7
\end{gathered}
$$

Since the above solutions are not (very) sensitive for changes in the pipe diameter $D_{p}$ or the line speed $v_{l s}$, but mainly for changes of the density ratio $\rho_{m} / \rho_{l}$, a comparison is made for a $D_{p}=1 \mathrm{~m}$ diameter pipe at a line speed of $v_{l s}=5 \mathrm{~m} / \mathrm{s}$ in sand with a solids density of $2.65 \mathrm{ton} / \mathrm{m}^{3}$ and a virtual solid with a density of $10 \mathrm{ton} / \mathrm{m}^{3}$. Fig. 9 and Fig. 10 show the Darcy-Weisbach friction factor ratios and the relative excess pressure gradient coefficient $\alpha_{E}$. The methods 2 and 4 without damping do not differ too much, both give a reduction on the solids effect of about $18-26 \%$ in sand for medium concentrations. The methods 1 and 3 with damping however give a reduction on the solids effect of about $55-65 \%$ in sand, almost 3 times as much. For the virtual solid with a solids density of $10 \mathrm{ton} / \mathrm{m}^{3}$, the reductions are $18-30 \%$ and $65-80 \%$. Based on the data as shown by Talmon (2013), the reduction of the solids effect of 55-65\% with method 1 with damping is overestimating the reduction, while the two methods without damping seem to underestimate the reduction, assuming that the reduction measured is caused by the effect of a lubricating viscous sub-layer.

If damping is added to the Nikuradse (1933) mixing length equation, method 3, the same results are obtained as the Prandtl mixing length equation with von Driest (Schlichting, 1968) damping, method 1. Apparently the mixing length damping dominates the difference between the 3 methods. The von Driest modification is an empirical damping function that fits experimental data, and also changes the near-wall asymptotic behavior of the eddy viscosity $v_{t}$, from $z^{2}$ to $z^{4}$. Although neither of them are correct (DNS-data gives $v_{t}$ proportional to $z^{3}$ ), the von Driest damping generally improves the predictions. It has, since its first appearance, repeatedly been used in turbulence models to introduce viscous effects in the near-wall region. The von Driest damping however has never been developed to deal with the problem of a lubricating viscous sub-layer as is elaborated in this chapter.

Because of the overestimation of methods 1 and 3 and the underestimation of methods 2 and 4 , an average between Prandtl without damping, method 3, and Prandtl with damping, method 1, could be used according to (with $\alpha_{h}$ about 6.7):

$$
\frac{\lambda_{m}}{\lambda_{l}}=\frac{1}{\left(\frac{\left(\frac{1}{\kappa} \cdot \ln \left(1+R_{s d} \cdot C_{v}\right)+\alpha_{h} \cdot R_{s d} \cdot C_{v}\right)}{2} \cdot \sqrt{\frac{\lambda_{l}}{8}}+1\right)^{2}}
$$




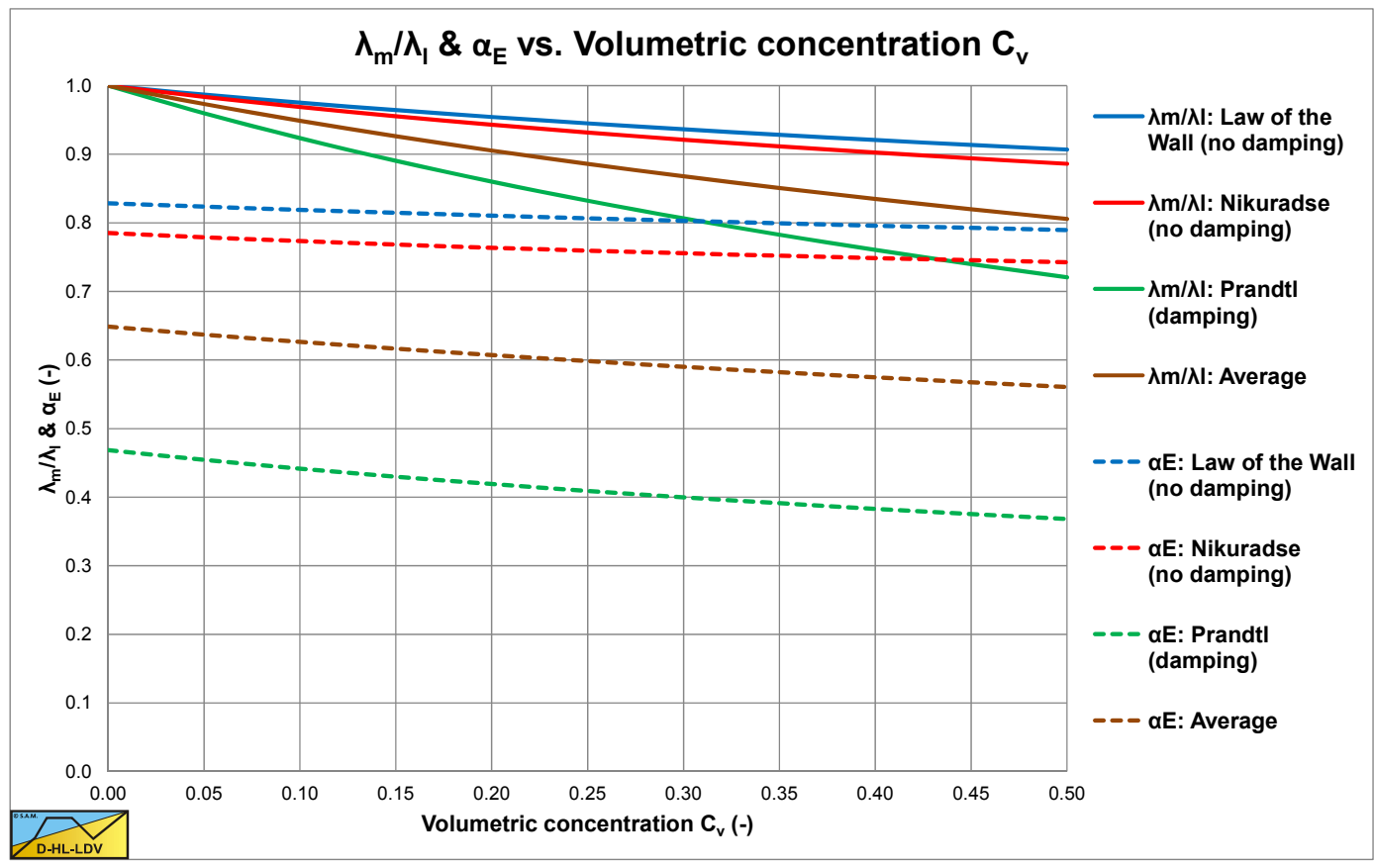

Fig. 9. The Darcy-Weisbach friction coefficient ratio $\lambda_{m} / \lambda_{l}$ and the factor $\alpha_{E}$ as a function of the volumetric concentration $C_{v}$ at $D_{p}=1 \mathrm{~m}, v_{l s}$ $=5 \mathrm{~m} / \mathrm{s}$ and solids density of $2.65 \mathrm{ton} / \mathrm{m}^{3}$.

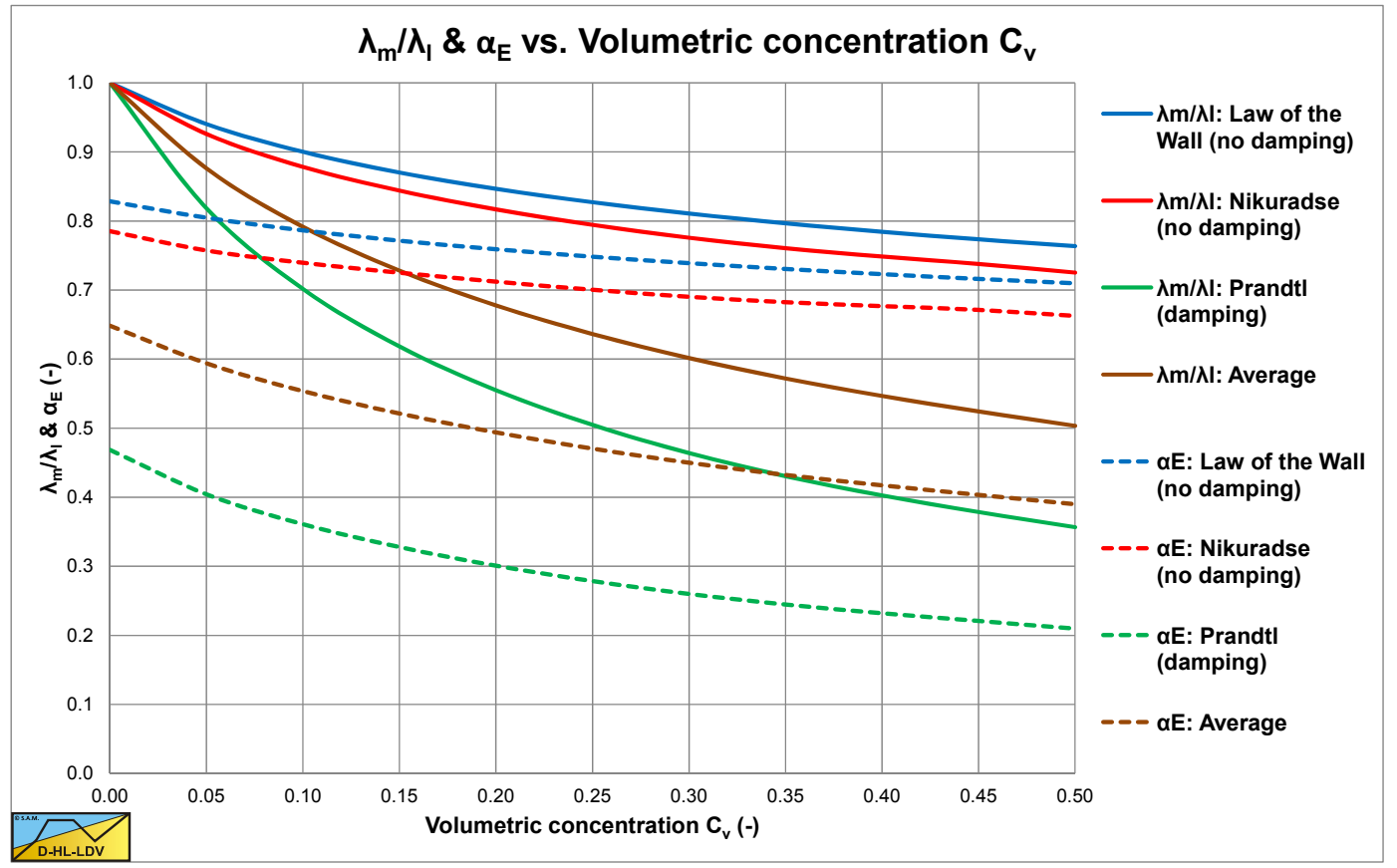

Fig. 10. The Darcy-Weisbach friction coefficient ratio $\lambda_{m} / \lambda_{l}$ and the factor $\alpha_{E}$ as a function of the volumetric concentration $C_{v}$ at $D_{p}=1 \mathrm{~m}$, $v_{l s}=5 \mathrm{~m} / \mathrm{s}$ and solids density of $10 \mathrm{ton} / \mathrm{m}^{3}$.

The results of this equation are also shown in Fig. 9 and Fig. 10. For sands with a solids density of $2.65 \mathrm{ton} / \mathrm{m}^{3}$ this gives a reduction of about $35-45 \%$, on average $40 \%$. The downside of this equation is, that the equation gives a fixed result for a fixed $R_{s d} \cdot C_{v}$ value and is not adaptable to more experimental data. Reason to investigate the possibility of applying a concentration profile, where the concentration equals zero at the pipe wall and increases, with a sort of von Driest damping function, to a maximum value at the center of the pipe. This concentration profile has to be corrected, based on numerical integration, to ensure that the average concentration matches a given value.

\section{METHOD 5: APPLYING A CONCENTRATION PROFILE TO METHOD 2}

The original Talmon (2013) concept assumes a constant density ratio for the whole cross section of the pipe. This of course is not in agreement with the physical reality. The concept assumes carrier liquid in the viscous sub-layer and mixture in the remaining part of the cross-section, but uses a constant density ratio. In order to correct this a damping factor for the density ratio $\alpha_{\rho}$ is proposed. This density ratio damping factor takes care that there is only carrier liquid very close to the wall. 
The factor $A_{\rho}$ determines the thickness of this carrier liquid layer. If $A_{\rho}$ equals zero, the solution obtained with Prandtl or Nikuradse with von Driest damping is found, methods 1 and 3. If $A_{\rho}$ equals 4.13 the solution of the "Law of the Wall" is found, method 4, and if $A_{\rho}$ equals 3.02 the solution of the Nikuradse equation without damping is found, method 2.

The concentration profile and the density ratio are defined as:

$C_{v, z}=C_{v, \max } \cdot\left(1-e^{-A_{\rho} \cdot \frac{z}{\delta}}\right)$

and $\quad \alpha_{\rho}=\frac{1+R_{s d} \cdot C_{v, z}}{1+R_{s d} \cdot C_{v}}$

The maximum concentration $C_{v, \max }$ in the concentration profile is found by integrating the concentration profile over the cross-section of the pipe and making it equal to the average concentration multiplied with the cross section of the pipe according to:

$$
\begin{aligned}
& \int_{0}^{R} C_{v, z} \cdot 2 \cdot \pi \cdot(R-z) \cdot d z \\
& =2 \cdot \pi \cdot C_{v, \max } \cdot \int_{0}^{R}\left(1-e^{-A_{\rho} \cdot \frac{z}{\delta}}\right) \cdot(R-z) \cdot d z \\
& =C_{v} \cdot \pi \cdot R^{2}
\end{aligned}
$$

The maximum concentration $C_{v, \max }$ is now equal to average concentration $C_{v}$ times a correction factor.

$$
\begin{aligned}
& C_{v, \max }= \\
& C_{v} \cdot \frac{\pi \cdot R^{2}}{2 \cdot \pi \cdot\left(\frac{R^{2}}{2}-\frac{R \cdot \delta}{A_{\rho}}+\left(\frac{\delta}{A_{\rho}}\right) \cdot\left(1-e^{-A_{\rho} \cdot \frac{R}{\delta}}\right)\right)}
\end{aligned}
$$

The velocity gradient, including the concentration profile, is now:

$$
\frac{\partial u}{\partial z}=
$$$$
\frac{-\alpha_{\rho} \cdot v_{l}+\sqrt{\left(\alpha_{\rho} \cdot v_{l}\right)^{2}+4 \cdot \ell^{2} \cdot\left(u^{*}\right)^{2} \cdot\left(\frac{R-z}{R}\right)}}{2 \cdot \ell^{2}}
$$

The integrated velocity difference $u_{m}-u_{l}$ is now:

$$
\begin{aligned}
& v_{l s, m}-v_{l s, l} \approx u_{m}-u_{l} \\
& =\int_{0}^{R} \frac{-\alpha_{\rho} \cdot v_{l}+\sqrt{\left(\alpha_{\rho} \cdot v_{l}\right)^{2}+4 \cdot \ell^{2} \cdot\left(u^{*}\right)^{2} \cdot\left(\frac{R-z}{R}\right)}}{2 \cdot \ell^{2}} \cdot d z \\
& -\int_{0}^{R} \frac{-v_{l}+\sqrt{\left(v_{l}\right)^{2}+4 \cdot \ell^{2} \cdot\left(u^{*}\right)^{2} \cdot\left(\frac{R-z}{R}\right)}}{2 \cdot \ell^{2}} \cdot d z
\end{aligned}
$$

For the resulting Darcy-Weisbach friction factor ratio this can be approximated by:

$$
\frac{\lambda_{m}}{\lambda_{l}}=\frac{1}{\left(\frac{A_{C_{v}}}{\kappa} \cdot \ln \left(\frac{\rho_{m}}{\rho_{l}}\right) \cdot \sqrt{\frac{\lambda_{l}}{8}}+1\right)^{2}}
$$

where $A_{C v}$ depends on the value of $A_{\rho}$. The relative excess hydraulic gradient $E_{r h g}$ is now:

$$
\begin{aligned}
& E_{r h g}= \frac{i_{m}-i_{l}}{R_{s d} \cdot C_{v}}=\alpha_{E} \cdot i_{l} \\
&=i_{l} \cdot \frac{1+R_{s d} \cdot C_{v}-\left(\frac{A_{C_{v}}}{\kappa} \cdot \ln \left(\frac{\rho_{m}}{\rho_{l}}\right) \cdot \sqrt{\frac{\lambda_{l}}{8}}+1\right)^{2}}{R_{s d} \cdot C_{v} \cdot\left(\frac{A_{C_{v}}}{\kappa} \cdot \ln \left(\frac{\rho_{m}}{\rho_{l}}\right) \cdot \sqrt{\frac{\lambda_{l}}{8}}+1\right)^{2}}
\end{aligned}
$$

Now, from numerical solutions, it appears that equations (36) and (37) give a very good approximation of all 4 methods for the range of parameters as normally used in dredging. The factor $A_{C v}=1$ for the "Law of the Wall" (method 4), $A_{C v}=1.25$ for the Nikuradse solution without damping (method 2) and $A_{C v}$ $=3.4$ for the Prandtl and Nikuradse solutions with von Driest damping (methods 1 and 3). The average equation (30) has a coefficient of $A_{C v}=2.2$ and $A_{\rho}=1.05$. Table 1 gives an overview of these values.

Table 1. Some $A_{\rho}$ and $A_{C v}$ values.

\begin{tabular}{lll}
\hline & $A_{\rho}$ & $A_{C_{v}}$ \\
\hline Law of the Wall & 4.13 & 1.00 \\
\hline Nikuradse (no damping) & 3.02 & 1.25 \\
\hline Prandtl (damping) & 0.01 & 3.40 \\
\hline Average & 1.05 & 2.20 \\
\hline Lower limit of data & 5.43 & 0.80 \\
\hline Upper limit of data & 1.67 & 1.80 \\
\hline
\end{tabular}

Fig. 11 shows a lower limit of the data, an upper limit of the data and the curve of Talmon (2013), method 1, compared with experimental data of Talmon (2011) in a vertical pipe. The lower and upper limit are determined for the particles from $d=$ $0.345 \mathrm{~mm}$ to $d=0.750 \mathrm{~mm}$. The finest particles of $d=0.125$ $\mathrm{mm}$ show less or even a reversed influence, probably because of the Thomas (1965) viscosity effect.

Fig. 1 shows experimental data of Thomas (1976) of iron ore in a horizontal pipe, where the theoretical curve contains both the Thomas (1965) viscosity and equation (37) with $A_{C v}=1.3$, the average of the lower and upper limit.

\section{CONCLUSIONS}

The concept of Talmon (2013) is applicable for determining the pressure losses in the homogeneous regime, however this concept has to be modified with respect to the shear stress distribution, the concentration distribution and a check on conservation of volume flow and concentration. The resulting equations (36) and (37) with $A_{C v}=1.3$ give a good average behavior based on the data of Talmon (2011) and Thomas (1976). The original factor $A_{C v}=3.4$ of Talmon (2013) seems to overestimate the reduction of the solids effect. It should be mentioned that the experiments as reported by Talmon (2011) were carried out in a vertical pipe ensuring symmetrical flow. 


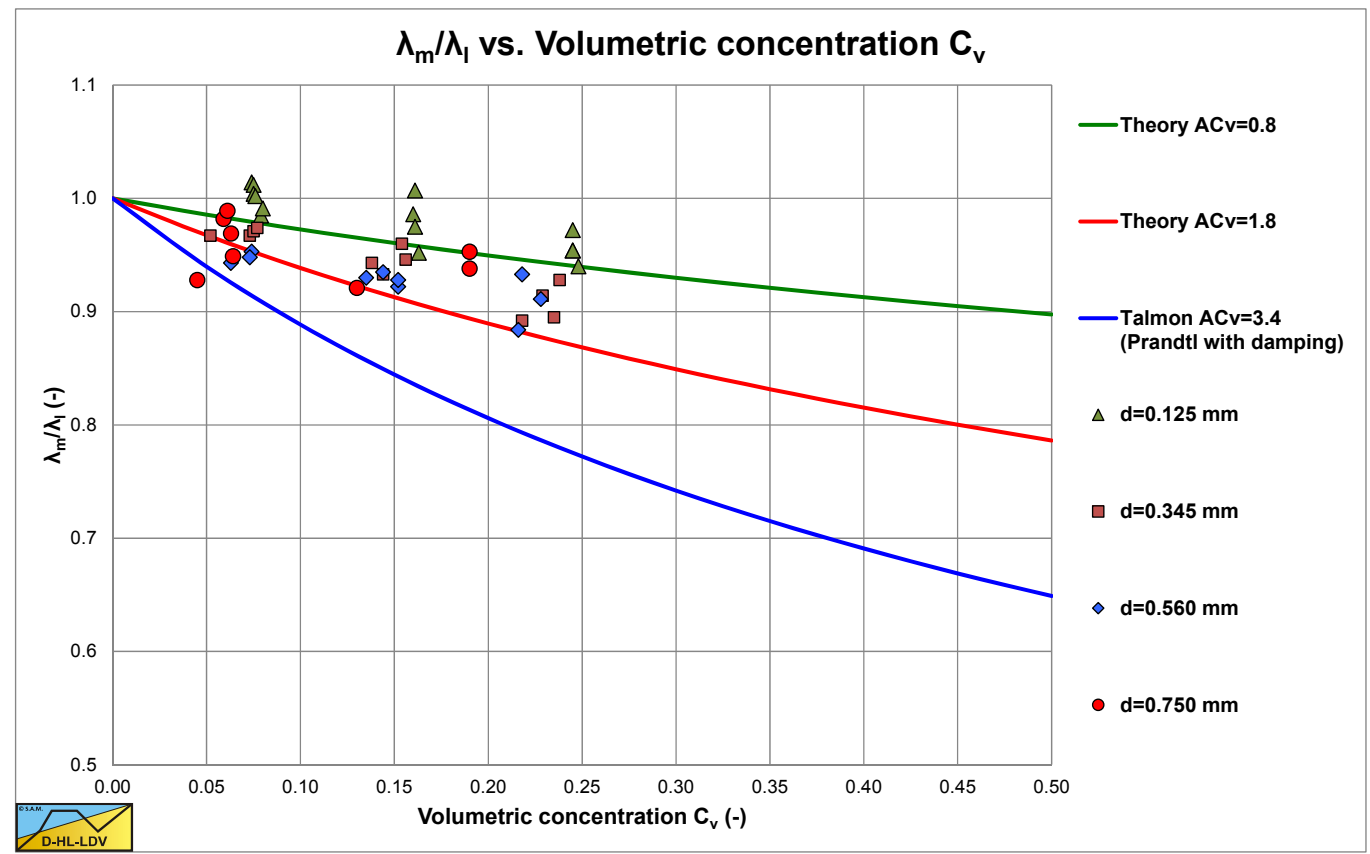

Fig. 11. Talmon (2011) data compared with the theory.

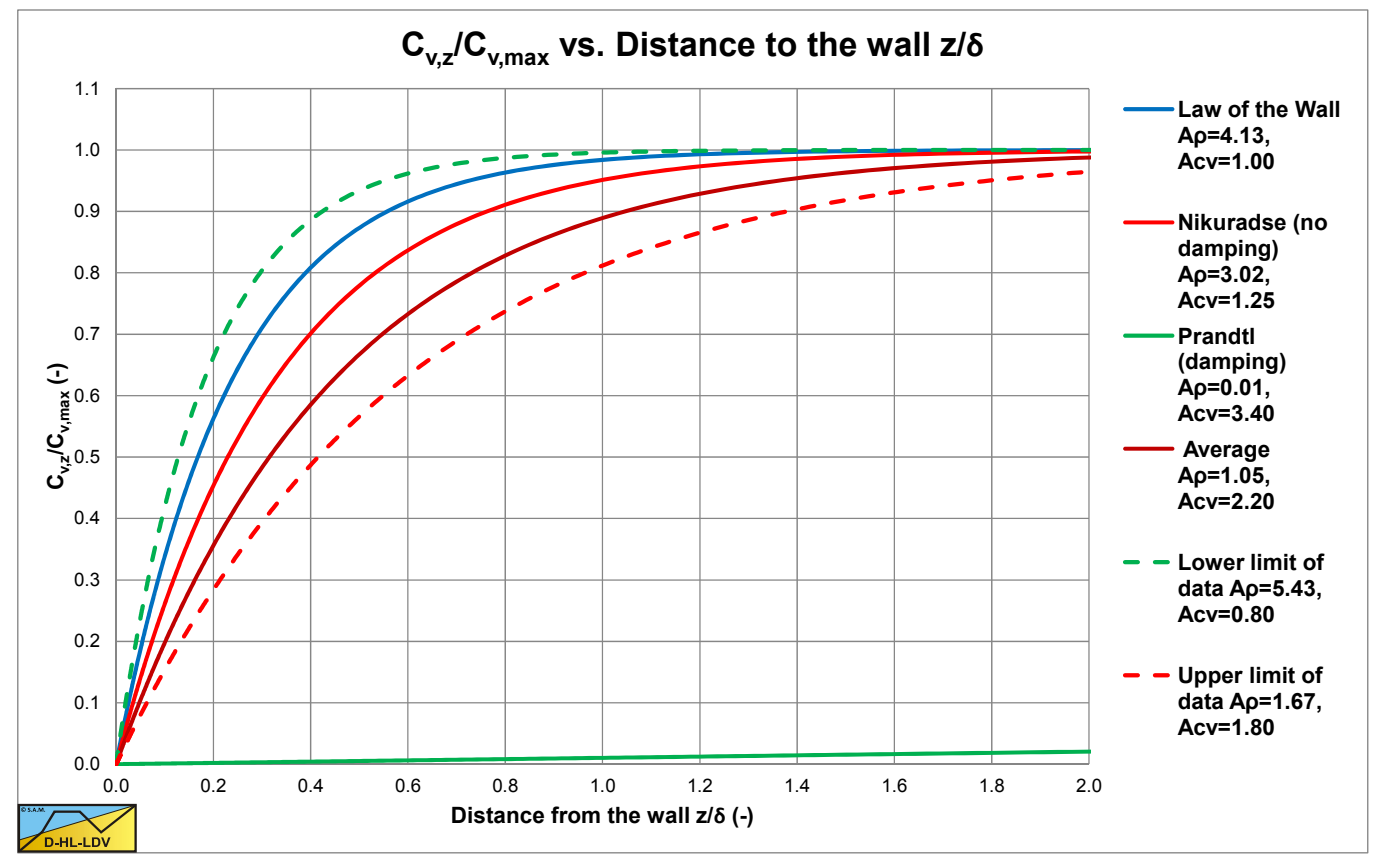

Fig. 12. The concentration distribution for the cases considered from Table $1 . D_{p}=1 \mathrm{~m}, v_{l s}=5 \mathrm{~m} / \mathrm{s}, \delta=0.088 \mathrm{~mm}$.

For horizontal pipes the results may differ, since the velocity and concentration profiles are not symmetrical at the line speeds common in dredging. The concentration profiles are shown in Figure 12. Since the model is based on a particle free viscous sub-layer and the viscosity of the carrier liquid, it may not give good predictions for very small or large particles. Very small particles may influence the viscosity, while very large particles are not influenced by the viscous sub-layer. Using the resulting equations (36) and (37), implies using von Driest damping in combination with a concentration profile. The resulting equations (36) and (37) are flexible in use.

The error of using $A_{C v}=1.3$ is difficult to define. With respect to the relative excess hydraulic gradient $E_{r h g}$ the accuracy is about $\pm 10 \%$. With respect to the hydraulic gradient $i_{m}$, which is of interest for the dredging companies, the accuracy is better to much better, since this hydraulic gradient equals $i_{m}=$ $i_{l}+E_{r h g} \cdot R_{s d} \cdot C_{v}$.

Equation (37) is implemented in the Delft Head Loss \& Limit Deposit Velocity (DHLLDV) model with a default value of $A_{C v}=1.3$ (see Miedema and Ramsdell (2014)).

\section{REFERENCES}

Miedema, S.A., Ramsdell, R.C., 2014. The Delft Head Loss \& Limit Deposit Velocity Model. Hydrotransport, BHR Group, Denver, USA, $15 \mathrm{p}$.

Newitt, D.M., Richardson, M.C., Abbott, M., Turtle, R.B., 1955. Hydraulic conveying of solids in horizontal pipes. 
Transactions of the Institution of Chemical Engineers, 33, 93 110.

Nikuradse, J., 1933. Stromungsgesetze in rauen Rohren. VDI Forschungsheft 361, Beilage zum "Forschung auf dem Gebiete des Ingenieurwesens", Ausgabe B, Band 4. (In German.)

Prandl, L., 1925. Bericht uber Untersuchungen zur ausgebildeten Turbulenz. Z. angew. Math. Mech., 5, 1, 136-139. (In German.)

Schlichting, H., 1968. Boundary Layer Theory. 6th ed. New York: McGraw-Hill.

Talmon, A., 2011. Hydraulic resistance of sand-water mixture flow in vertical pipes. In: Transport and Sedimentation of Solid Particles. T\&S, Wroclaw, Poland, pp. 137-147.

Talmon, A., 2013. Analytical model for pipe wall friction of pseudo homogeneous sand slurries. Particulate Science \& Technology, 31, 3, 264-270.

Thomas, A., 1976. Scale-up methods for pipeline transport of slurries. International Journal of Mineral Processing, 3, 51-69.

Thomas, D.G., 1965. Transport characteristics of suspensions: VIII. A note on the viscosity of Newtonian suspensions of uniform spherical particles. Journal of Colloidal Sciences, 20, 267-277.

Wilson, K.C., Addie, G.R., Clift, R., 1992. Slurry Transport using Centrifugal Pumps. Elsevier Applied Sciences, New York.

\section{NOMENCLATURE}

\begin{tabular}{|c|c|c|}
\hline$A$ & Von Driest damping factor (26) & - \\
\hline$C v$ & Concentration factor & - \\
\hline$A_{\rho}$ & Density factor & - \\
\hline$C_{v}$ & $\begin{array}{l}\text { Concentration averaged over the cross } \\
\text { section of the pipe }\end{array}$ & - \\
\hline$C_{v, z}$ & Concentration at distance $\mathrm{z}$ of the pipe wall & - \\
\hline$C C_{v, \max }$ & $\begin{array}{l}\text { Maximum concentration in the center of } \\
\text { the pipe }\end{array}$ & - \\
\hline$D_{p}$ & Pipe diameter & $\mathrm{m}$ \\
\hline$E_{r h g}$ & Relative excess hydraulic gradient & - \\
\hline$F$ & Homogeneous reduction factor & - \\
\hline$g$ & Gravitational constant $(9.81)$ & $\mathrm{m} / \mathrm{s}^{2}$ \\
\hline$\Delta L$ & Length of pipe segment considered & $\mathrm{m}$ \\
\hline$i_{m}$ & Mixture hydraulic gradient & - \\
\hline$i_{l}$ & Liquid hydraulic gradient & - \\
\hline$R$ & Pipe radius & $\mathrm{m}$ \\
\hline$R_{s d}$ & Relative submerged density (sand 1.65) & - \\
\hline$u$ & Velocity & $\mathrm{m} / \mathrm{s}$ \\
\hline$u_{l}$ & Velocity liquid & $\mathrm{m} / \mathrm{s}$ \\
\hline$u_{m}$ & Velocity mixture & $\mathrm{m} / \mathrm{s}$ \\
\hline$u_{*}$ & Friction velocity & $\mathrm{m} / \mathrm{s}$ \\
\hline$v_{l s}$ & Line speed & $\mathrm{m} / \mathrm{s}$ \\
\hline$v_{l s, l}$ & Line speed liquid & $\mathrm{m} / \mathrm{s}$ \\
\hline$v_{l s, m}$ & Line speed mixture & $\mathrm{m} / \mathrm{s}$ \\
\hline$z$ & Distance to the wall & $\mathrm{m}$ \\
\hline$\alpha_{h}$ & Homogeneous factor & - \\
\hline$\alpha_{\text {Erhg }}$ & Homogeneous factor $\mathrm{E}_{\mathrm{pg}}$ value & - \\
\hline$\beta$ & Nikuradse correction factor & - \\
\hline$\lambda_{l}$ & Darcy-Weisbach friction factor liquid & - \\
\hline$\lambda_{m}$ & Darcy-Weisbach friction factor mixture & - \\
\hline$\rho_{l}$ & Density liquid & ton $/ \mathrm{m}^{3}$ \\
\hline$\rho_{m}$ & Density mixture & ton $/ \mathrm{m}^{3}$ \\
\hline$\kappa$ & Von Karman constant (0.4) & - \\
\hline$\tau$ & Shear stress & $\mathrm{kPa}$ \\
\hline$\tau_{v}$ & Viscous shear stress & $\mathrm{kPa}$ \\
\hline$\tau_{t}$ & Turbulent shear stress & $\mathrm{kPa}$ \\
\hline$\mu_{v}$ & Viscous dynamic viscosity & $\mathrm{Pa} \cdot \mathrm{s}$ \\
\hline$\mu_{t}$ & Turbulent dynamic viscosity & $\mathrm{Pa} \cdot \mathrm{s}$ \\
\hline$\mu_{l}$ & Dynamic viscosity liquid & $\mathrm{Pa} \cdot \mathrm{s}$ \\
\hline$\mu_{m}$ & Dynamic viscosity mixture & $\mathrm{Pa} \cdot \mathrm{s}$ \\
\hline$v_{l}$ & Kinematic viscosity liquid & $\mathrm{m}^{2} / \mathrm{s}$ \\
\hline$v_{m}$ & Kinematic viscosity mixture & $\mathrm{m}^{2} / \mathrm{s}$ \\
\hline$v_{t}$ & Turbulence viscosity & $\mathrm{m}^{2} / \mathrm{s}$ \\
\hline$\ell$ & Mixing length & $\mathrm{m}$ \\
\hline
\end{tabular}

Received 3 March 2014 Accepted 8 September 2014 